\title{
Mesure du débit des oueds nord-africains par la méthode chimique au bichromate
}

\author{
Application aux oueds de Kabylie des Babors et des hauts plateaux sétifiens \\ Discharge measurements of North African waddis \\ by the chemical bichromate method
}

\author{
Application to the waddis of Kabylie and the high Setif plateaux
}

\author{
A.-A. GUNT/

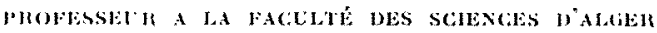

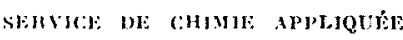

PAR

F.T

\author{
A. LEXO

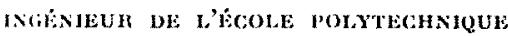

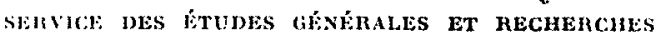 \\ D'ÉLECTHGTE ET GAZ D'ALGÉ
}

\begin{abstract}
Les auteurs ont expérimenté la méthode de jaugeage chimique au bichromate de sodium (methode Dodero) en y apportant une modification appropriée aux eau. $x^{2}$ troubles susceptibles de réduire le bichromate.

Laddition d'un peu de permanganate aux prilevements faits en me dn dosage stabilise le bichromate ef la technique du dosage colorimétrique reste utilisable moyennant quelques petites modificutions.

Les résultats obtenus confirment la sensibilité et la précision de la méthode chimique Dodero; ils permettent de l'appliquer sans crainte à.des eaux suspectes, susceptibles de perturber les résultats par rédluction du bichromate.
\end{abstract}

\begin{abstract}
The authors have carried out experiments on the chemical gauging method using sodium bichromate (Dadero method) and have modified it to suit the turbid waters which are likely to reduce the bichromate.

The addition of a little permanganate to the samples is made immediately to stabilise the bichromate: and the technique of calorimetric dosage remains useable provided a few small modifications are made.

The results oblained confirm the sensitivily and accuracy of the Hodero chemical method; they show that the method can be applied with certainty to doubtful waters, which at first secm likely to upset the results by reducing the bichromate.
\end{abstract}

En pratique, il faut trouver un produit chimique qui cumule un certain nombre de qualités :

1" Etre facile a se procurer à un prix abordable;

2" Etre facilement soluble, ni corrosif, ni toxique;

3" Etre facile a doser avec précision, même aux Irès grandes dilutions (de l'ordre du mg/litre);

t $^{\circ}$ Ve pas préexister en quantite comparable dans leall du eours d'ean; 
$5^{\circ}$ Ne pas se détruire ou disparaître entre le moment de l'injection et l'analyse du prélèvement.

Ces conditions restreignent naturellement beaucoup le choix possible.

Aussi le simple sel marin, facile à doser et proposé à l'origine par Schloesing (1), instigateur de la méthode chimique, n'est pas applicable aux eaux de rivières tant soit peu salées, comme le sont précisément les oueds nord-africains.

La fluoresccine, naturellement absente, est détruite par la lumiere et son dosage précis n'est pas facile, aussi trouve-t-elle un champ d'application limité aux eaux souterraines.

Finalement, un petit nombre de composés chimiques ont franchi le batrage des conditions nécessaires. Ce sont :

-.- le sulfate de manganèse.

-. le nitrite de sodium,

- le bichromate de sodium, dernier venu dont la qualité majeure est la sensibilité du dosage à la diphényl-carbazide. Absent dans les eaux naturelles et, sous certaines conditions, stable, le bichromate doit être plus avantageux que le nitrite de sodium dont le dosage a une sensibilité du même ordre.

L'emploi d'isotopes radioactifs, susceptibles d'ètre dètectés par la mesure physique du rayonnement caractéristique, est très probablement une solution nouvelle, solution d'avenir que nous ne pouvions envisager avec les moyens dont disposent les chimistes.

Le Service des Etudes Générales et Recherches d'Electricité et Gar d'Algérie a en projet des aménagements hydro-électriques importants sur l'Oued Bou-Sellam ef l'Oued Djendjen. Les conditions des mesures sont telles que les jaugeages au moulinet s'avèrent difficiles et imprécis; la méthode chimique de mesure des débits a donc été mise en cuvre en utilisant le bichromate.

Il est rapidement apparu que le bichromate n'avait pas la stabilité prévue. Les eaux d'oueds, très chargées en sédiments et en matières organiques, deviennent susceptibles de réduire le bichromate.

Il est très plausible d'admettre une réduction biochimique et l'un de nous a pensé stopper ce processus en ajoutant, sitôt le prélèvement fait, une petite quantité de permanganate de potassium jouant à la fois le rôle d'antiseptique, d'oxydant protecteur et même régénérateur du bichromate éventuellement réduit.

La technique du dosage se présente dès lors ainsi :
- Aussitòt faits, les prélèvements sont additionnés de quelques gouttes d'une solution de permanganate de potassium concentrée, - leinte obtenue : vin rosé;

Si les échantillons doivent ètre adressés à un laboratoire distant el si quelques délais sont à prévoir, les flacons seront de préférence bouchés à l'émeri ou en matière plastique.

A l'arrivée au laboratoire, on procède à la décoloration au moyen d'azoture de sodium. Il faut en ajouter le moins possible - - soit quelques gontles d'une solution at $1 \%$, soit quelques cristaux du sel solide. On opere par additions successives en suivant la décoloration qui est très rapide au bain-marie, quinze minutes au plus. Aussitòt décolorie, la solution est refroidie. Dans ces conditions, le permanganate est réduit sans que le chromate soit touché et sans que soit gênée la réaction à la diphénylcarbazide (6).

L'expérience révèle facilement les conditions susceptibles de donner la sensibilité la meilleure avec le colorimetre dont on dispose et les concentrations des prélèvements. Quoique la loi de BEER soit sensiblement suivie (filtre vert - $540 \mathrm{m \mu}$. pour le maximum d'absorption), il est toujours convenable de tracer une courbe expérimentale avec des quantités connues de chromate et les mêmes réactifs.

L'expérience montrera si une addition très faible de permanganate suivie de décoloration spontance est suffisante à l'usage.

\section{I. - Mesure de débits de résurgences DANS L'OUED BOU-SELLAM}

L'Oued Bou-Sellam traverse au nord-ouest de Sétif un massif calcaire où il s'encaisse en gorges profondes (gorges du Guergour); la question s'est posée de mesurer le débit des résurgences situées dans le lit de l'oued.

Les débits, de 200 à $600 \mathrm{l} / \mathrm{s}$, ont été mesurés au moyen d'un équipement très léger. Le tronçon de l'oued en régime torrentiel sur lequel ont porté les essais était choisi juste assez long pour que la dilution de la solution soit bien homogène. Les prélèvements ont été effectués jusqu'à la teneur nulle. Le débit de l'oued, pratiquement constant pendant les mesures, a été calculé en écrivant que le poids total de bichromate injecté se retrouve dans la section de prélèvement. Le traitement au permanganate a été effectué au moment du flaconnage, bien que les eaux fussent claires, du fait de la présence possible de corps organiques et des très faibles concentrations à doser vers la fin de l'opération. 


\section{I1. - MESTRES DE DEBBITS DE L'OUED DJENDJEN}

L'Oued Djendjen à la station de mesure draine un bassin versanl de $133 \mathrm{~km}^{2}$, qui comprend un des plus hauts sommets de la Kabylie des Babors (Djebel Tababor $1.960 \mathrm{~m}$ ); il est constitué par des schistes de perméabilité très faible, on y observe des crues violentes de $300 \mathrm{~m}^{3} / \mathrm{s}$ et audelà. Dès que le débit dépasse $10 \mathrm{~m}^{3} / \mathrm{s}$, il esl très difficile de maintenir un moulinet aux points voulus d'une verticale déterminée de la section et la mesure de hauteur d'eau ne peut être faite avec précision.

Ces raisons ont conduit it rejeter les jaugeages au moulinel pour les débits supérieurs à $10 \mathrm{~m}^{3} / \mathrm{s}$.

La méthode de jaugeage chimique donnera par contre une valeur moyenne du débit plus exacte, sans qu'il soit nécessaire de connaitre avec précision la section d'écoulement.

L'équipement de jaugeage, fixe, a été conçu pour les débits jusqu'à $300 \mathrm{~m}^{3 /} / \mathrm{s}$, il est analogur à celui décrit par $H$. Dumas (5).

L'injection est faite en 1,2 ou 3 points de la section (débil réglable a partir de la rive); le mélange est effectué sur une longueur d'oued de $800 \mathrm{~m}$, avec deux coudes de sens inverse.

Cependant, nous avons vu que le bichromate pouvait être réduit par les eaux; il faut contròler que la quantité de bichromate injectée est bien retrouvée dans la section de prélèvement; on a donc effectué les prélèvements nécessaires pour tracer la courbe complete de variation de la concentration dans l'oued jusqu'aux concentrations nulles.

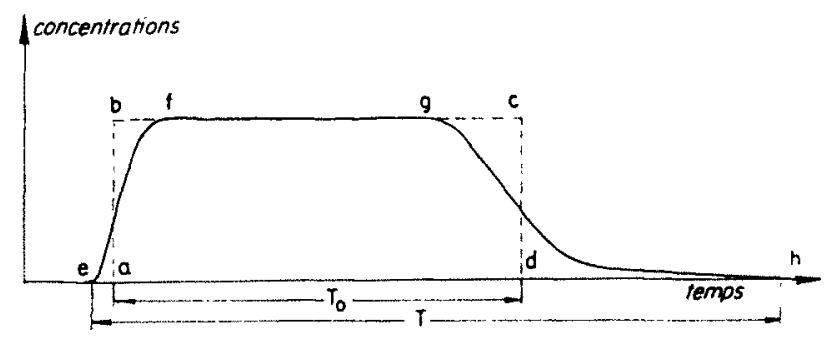

Soit :

Q le débit de l'oued,

$q$ le débit d'injection,

C la concentration de la solution injecté,

c la concentration en régime permanent,

$c_{i}$ la concentration, à l'instant $i$,

$n$ le nombre de prélèvements,

To la durée d'injection,

$T$ la durée des prélèvements de $C_{11}=0$ à la concentration finale de nouveau nulle $C_{p}=0$.
Lorsque le bichromate injeclé n'a pas été réduit, les surfaces abcd et efgh sont égiles, et :

$$
\mathrm{v}\left(c_{i} / n\right) \times \mathrm{T}=r \mathrm{~T} \%
$$

Le rapport du poids total de bichromate injecté à la somme dans le temps des concentrations des prélèvements permet aussi l'évaluation du débit moyen $Q$ pendant l'opération -.. ce débit sera égal au débil de régime permanenl $\mathrm{Q}=\mathrm{Cq} / \mathrm{e}$ s'il n'y a pas de disparition de bichromate.

Nous donnons ci-dessous les resultats d'un des jaugeages effectué dans ces conditions.

\section{Jaugeage effectué le 18 mars 1954}

Débit d'injection : $0,4461 / \mathrm{s}$;

Durée d'injection : 1 heure;

Teneur en $\mathrm{Cr}_{2} \mathrm{O}_{7} \mathrm{Na}_{2}, 2 \mathrm{H}_{2} \mathrm{O}=22 \mathrm{gr} / 1$;

beux points d'injection dans le profil de $50 \mathrm{~m}$ de large, débits égaux;

Prélèvements : toutes les deux minutes pendant deux heures. Simultanément en trois points du profil en travers de la section de prélevements à $800 \mathrm{~m}$ en aval des points d'injection;

Nombre de prélévements : 180 ;

Traitement au permanganate dis flaconnage.

Concentration moyenne des prélevements: $0,937 \mathrm{mgr} / \mathrm{l}$.

Débit de loued (régime permanent) :

$$
Q=0,446 \frac{22}{0,937}=10,17 \mathrm{~m}^{2} / \mathrm{s}
$$

Débit de louled contrôle):

$$
\mathrm{Q}=\frac{3.600 \times 22 \times 0.446+836}{3.480 .72}=10,37 \mathrm{~m}^{3} / \mathrm{s}
$$

Le lerme 836 est une correction de vidange les tubes.

\section{Remarques}

(1) Lo jaugeage au moulinel encore possible à ce débit, effectué dans la section de prélivement, a donné :

$$
Q=10,4 \mathrm{~m}^{3} / \mathrm{s}
$$

en tres bonne concordance avec les résultats précédents -... différence inférieure a $1 \%$; 
b) Les eaux de l'oued itaient troubles et chargées à $1 \mathrm{gr} / \mathrm{l}$ de matières en suspension. L’un de nous (7) a déjà signalé la réduction possible du bichromate dans ces conditions; l'erreur sur lo débil, qui pourail atteindre $30 \%$ par excès, a pu ĉtre ivitée par le traitement au permanganate, qui s'avere essentiel partout où des eaux pollućes ou chargées sont en cause.

\section{Conclusion}

1)ans les deux exemples cités, l'efficacité du permanganale a été rérifice: on a pu ainsi réaliser des jaugeages chimiques en eaux chargées avec la même précision qu'en eau très pure. Les mesures de débits des grandes crues pourront citre entreprises en loule scicurité.

\section{BIBLIOGRAPHIE}

(1) Schloksing (Th.). - C. R. Ac. des Sc., 1863.

(2) Gocues (J.). - « Jaugeage continu du Guil $»$, la Houille Blanche, $\mathrm{n}^{n} 3,1946$.

(3) Sociéte Hydrotechnique de France, fascicule $n^{\prime \prime} 4$ du «Code d'Essais des Installations Hydrauliques », article A II.

(4) Doneno. - C. R. Ac. des Sc., 243, 1462-t, 1952.

(5) Dumas (H.) . ...- La Honille Blanche, 690-701, 1952; $51-57,1953 ; 360-373,1953$.

(5 bis) Znnule (H), -- La Honille Blanche, ,510-4, 1953.

(6) Saltzmax (Bemard-les. Analytical chemistry. 1066,1952

(7) Gent (A.A.) - . C. K. Ac. des Se, 236, 2423-4, 1953.

(8) Doweno. - Modalités pour fe titrage des solutions cmploves dans la méthode chimique pour la mesure des debits $\$$. La Honille Blanche, no 6, 1953 .

\section{I S CUSSION}

Président : M. Rwvexwras

M. le Présidenl remercie Il Drmas d'aroir bien voulu présenter le mémoire de MM. (ivnr\% et Luso et félicile los atuteurs.

Répondant a une question de M. Brac sur l'homogenéité de la solution, M. Dumas indique que les auteurs ne donnent que la concentration finale moyenue sans préciser les concentrations correspondant a chaque point de prélèvement.

Mais M. Druas pense que celte concentration devait ètre homogène au bout du pareours de 800 metres considéré sur les oueds, car il a lui-mème obtenu une dilution homogène a moins de 1 "to dans la section inale d'un parcours de $500 \mathrm{~m}$ sur la Severaisse. Dans l'un et lautre cas, la turbulence a relativement grosse echelle et le faible tirant d'eau des cours deau étudies finissent par donner une bonne homogénéité. En ce qui concerne les coulements calmes, II. Dustas signale qu'il a operé dans le eanal du Drac infericur, a Grenoble, qui a $48 \mathrm{~m}^{2}$ de section et un alignement droit de $1 \mathrm{~km}$ de longureur : pour un débit de $60 \mathrm{~m}^{3} / \mathrm{s}$ correspondant a une vitesse de pius d'un mètre à la seconde, avec une injection répartie an surface au moven d'un tube perforé tous les 20 centimètres, il a obtenu, au bout d'un parcours de 400 mètres, une repartition assez homogène pour permettre un bon calcul du débit.

En genćral, les comparaisons avec d'autres methodes de jaugeage sont rares, ear là où la méthode ehimique s'applique telle autre ne s'applique plus, mais les statistiques ont montré que les présomptions d'exactitude de cette méthode sont bonnes.

M. Matinis demande comment est détermine le nombre de prélèvements suecessifs opérés en un même point de la section finale. Ce nombre est-il fixé suivant ha précision que l'on désire obtenir?

M. Dumas repond qu'on cherche à obtenir un regime permanent, et que ce régime permanent doit être assez long pour permettre un nombre de prélèvements suffisants pour tracer le palier de ce régime.

M. le Président rappelle quil y a cependant dans de mémoire de MM. Guntz et l.rou une comparaison ave des mesures au moulinet. L'écart inférieur a 1 ir qui en resulterait est, d'ailleurs, assez exceptionnel et mériterait une analyse particulière.

I. Bovvand indique qu'une corrêlation extremement satisfaisante a éte trouvée entre des mesures fates par M. DUmas et la R.E.H. Alpes II suivant la méthode ehimique et des mesures faites au moulinet par la Division rechnique Générale dW.D.F sur l'Arveyron a Chamonix. Far contre, à coté d'un très grand nombre d'essais différents, il a eté trouvé dans deux cas des anomalies diffidilement explicables :

$\rightarrow$ Sur le torrent de Naves, afluent rive droite de l'lsère où le jaugeage portait sur plusieurs kilomètres do torrent pour des débits de l'ordre de $100 \mathrm{l} / \mathrm{s}$ (il sagissait de mesurer les apports intermédiaires): dans ce eas on a trouvé une diversenec entre les debits indiques par jaugeases conlinus el les débits indiques par des jaugeages faits a lamont el i l'aval du troncon considere. Ces anomalies semblent dues it la plesence dans l'eau d'amats de branches of de feuilles.

-.-. Sur l'Arve au droil des Houches ou les anomalies semblent correspondre soit is la teneur elevio en matiere minerale (il sagissait de jaugeages des purges et des dessableurs? soil a la teneur en matières organiques blevé due a la présence de Chamonix i l'amont.

II. Bumas croit que la stabilite du bichromate en pré sence des matieres organiques est bonne, mais que ce sont les micro-organismes qui provoquent la reduction de ec sel. Des essais de stabilite du bichromate seront faits ti presence des caux recueillies dans les endroits signales par M. Bowykd dans des mares stagnantes.

Lorsque les caux présentent un trouble persistant dù la suspension ou a la dilution de produits minéraux, il suffit d'avoir soin de prélever dans la rivière même, la quantité d'eau nécessaire à la préparation de l'échelle de dilution servant à l'étalonnage du colorimètre.

Répondant ì une question de M. Ravier, M. Dumas dit yu'il ignore si l'emploi de l'azoture de sodium, qui a l'inconvénient d'être très toxique, présente comme antiseptique des avantages sur les solutions sulfuriques. Il ajoute que cortains chercheurs ont cherche a utiliser un 
antiseptique ou un procéde plysique ne proroquant pas de coloration : par exemple l'irradiation des echantillons sous une lampe a vapeur de mereure.

M. Raven domne les résultats de ses essais comparatifs portant sur les jaugeages a moulind, la mothode ohiinique et la méthode des résistivilés: dans les eatux de grande pureté ef de fable salinite telles que celles des torrents de monlagne, les résultals des frois méthodes concordent a $3 \%$ près; dans le Gave de Pau, pour des déoits de 60 à $80 \mathrm{~m} 3 / \mathrm{s}$, entre la méthode dess résistivités opérée avec des concentrations importantes de sel gemme (d'ailleurs moins soluble que le sel marin) et ha methode chimique, mise en weurre avec des teneurs de bichromate inférieures au milligramme, la concordance a été à peu près normale, inféricure à $5 \%$, par contre, les écarts avec les mesures au moulinet ont atteint 10 is $12 \%$

M. Ie Président remarque que lorsquon compare les résultats des jaugeages au moulinet arec ceux donnés par d'autres methodes, les erreurs propres à chacume d'elles penvent, suivant le hasard, s'ajouter ou se retrancher; on ne peut done tirer de conclusion qu'aperes des essais répétés.

N.II.T.R. ... Nous avons communiøue le texte de cetle discussion a Ml. Lexo qui nous a fait part des observalion ci-apres:

1) Au sujet de loppinion énise sur l'ecart inlericur a 1 entre les mesures de debit au moulinet el par la méthode chimique, effectues par les auleurs:

La mesure du débit a été effeetuce au moulinet en 57 points de la section de prelevements, sur te bedrock leve avee précision; en chaque point la mesure était effectuce sur une durée dian moins deux minutes. D'autre part si la répartition des coarts de mesures d'une mène grandeur par differents procedes est gaussienne les valeurs voisines Ite la moyenne, ici zéro, ont la probabilita maximum et derraient done etro les moins rares, ains il n'y a pas lieu de s'inquiefer outre mesule de la concordance observed a $1 \%$ pres environ.

2) Liaroture de sodium n'est cmploye quau haboraloire et sculement dentuellement pour decolorer le permanganate sal toxicile ne pose donc pas de problème.

3 Les anleurs ont adopte le permanganate paree que sa coulcur rébise automatiquement fe controle de son addition.

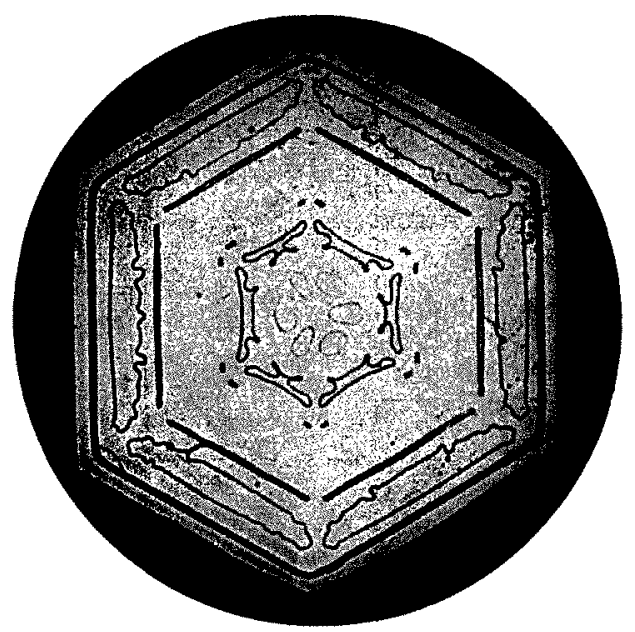

